\title{
Face Recognition System Based on Deep Residual Network
}

\author{
Juan Liang ${ }^{1, \mathrm{a}}$, Haoyu Zhao ${ }^{2, \mathrm{~b}}$, Xingqian $\mathrm{Li}^{1, \mathrm{c}}$ and Hongwei Zhao ${ }^{1, \mathrm{~d}}$ \\ ${ }^{1}$ College of Computer Science and Technology, JILIN University, Chang Chun130012, China \\ ${ }^{2}$ Editorial Department of Journal, JILIN University, Chang Chun130012, China \\ a liangjuan1105@163.com, bzhaohaoyu@jlu.edu.cn, ${ }^{\mathrm{c}}$ 2247755035@qq.com, ${ }^{\mathrm{d}}$ zhaohw@jlu.edu.cn
}

Keywords: Residual Network, Face Recognition, Android, Python, SL4A

\begin{abstract}
With the development of social science and technology, people's awareness of the safety of life gradually high, face recognition technology has entered people's lives .This paper designs a face recognition system based on the depth residual network.This system is implemented in both PC and Android. the PC terminal can add voice broadcast function, timely and effectively remind and prevent should be adopted when " danger" appears.The implementation of the Android mobile terminal is achieved by combining the Android and Python harmoniously through the platform of the Python scripting language provided by SL4A.Face recognition in the Android mobile side can be used for identity security authentication, mobile payment and other convenient operation, greatly increased the ease of operation of the system to make up for the PC side to carry inconvenient enough, the experiment proved that the face recognition technology Embedded into the Android terminal has a good recognition effect.
\end{abstract}

\section{Introduction}

In recent years, the application of face recognition is prevalent in life, especially in places where traffic is high and high in security requirements, such as banks, airports, railway stations, public security departments, hotels, car systems[1], laboratories and other places to identify, in order to protect the comfort of life and travel safety.

Face recognition technology has been widely used in domestic life, such as the 2008 Beijing Olympic games to apply face recognition technology in the opening and closing ceremonies, this feat is called the face recognition technology in China's development milestone[2].In 2015, Ma in the Cebit show "brush face payment", is Alipay face recognition technology, July 8, 2017, Ma's "unmanned supermarket[3]" opened, using the RFID + face recognition, Real-time supervision of the user's behavior, people's recognition of face recognition has also been greatly improved, mankind officially entered the "brush face" era.

This paper proposes a face recognition based on the depth residual network, which is proposed by He k[4]in 2015 and uses a 152 - layer imagenet data set to evaluate the network residual,The most notable feature of the network is that compared to some other Convolution neural network has a deeper network architecture (8 times than the VGG network), and has more than $96 \%$ accuracy and good convergence performance[5].The system is mainly written in Python, and the face recognition based on the residual network framework is invoked by using the Python interface provided by Dlib.In the PC terminal,the face is captured by the camera,and the final result of the way the voice broadcast to inform the user identification results, making the identification more intuitive, if the face recognition system for security detection can also give some potential security risks to remind.In the Android terminal, using SL4A[6] to provide the implementation of the combination of Python and Android,will be transplanted to the Android side at the PC side, complete the implementation of the mobile end, the implementation of the Android intelligent terminal provides guidelines for the application and promotion of face recognition in various industries. 


\section{System Overview}

The implementation of the system is mainly based on the Deep Residual Network, the implementation of the Android side is the use of sl4a to complete python writing Android applications.

\subsection{Residual Network}

This paper is mainly based on the depth of residual network to achieve face recognition, the reason why the use of the model to identify, is due to the overall residual network reached $3.57 \%$ error[4], in addition there are some advantages of residual network:

(1)The residual network is deep enough (up to 34 layers), which facilitates the extraction of more abstract, complex and easily distinguishable features, and can also be used to fit more complex mapping functions.

(2)The residual network is all convolution layer, non full connection layer, the convolution kernel is the convolution of $3 \times 3$ in size, which ensure that the network parameters as small as possible, making the network in a single limited computing power The GPU on the training, testing can be feasible.

(3)With the deepening of the depth,some of the problems come with it, such as the gradient explosion or disappearance occurs and the accuracy decreases.However, these problems are solved by the intermediate normalization and residual learning. Finally, the model not only deep depth but also high precision is obtained.

\subsection{SL4A}

By using the principle of SL4A, this article implements the Android program written in python.SL4A[6] full name for Scripting Layer for Android, is a project initiated by Google, as the Android script architecture layer,it works on the basis of RPC remote agent, the local script function package to pass to the remote Android Server APK, the final operation of the results of the feedback to the local script parser, and then show the results in the terminal[6].In essence, the implementation of this article on the Android side is the PC side of the implementation of the Android side of the embedded, achieve the perfect integration of Android and python, eliminating the need for masses of work in the Android.

\subsection{Design of $\mathrm{PC}$ and Android}

In this paper, the realization of face recognition is mainly through the Dlib provided by the Python interface for face detector, face key detector, depth residual network face recognition model to complete a series of image preprocessing, feature description sub-extraction and other face Identify work.

On the PC side, a face recognition server application is implemented, the use of ordinary USB camera to obtain the dynamic scene,camera open is to use the VideoCapture() function in OpenCV,and it captures and saves the image in the camera, setting the image every $5 \mathrm{~s}$ to capture the image, and with the candidate['Zhao Hongwei', 'Li Xinggan', 'Puppy Song Yifan' , "Jiang Youliang", 'Zhai Hong', 'Xu Xiaoli', 'Liang Juan']successively to count the European distance, The smaller the value, the more likely the match will be, so choose the minimum European distance value match the value is compared with the set precision value, and if match <accuracy, it is correct. The final recognition result is broadcast by the sound connected to the computer, making the recognition result more intuitive and understandable.

Android platform design, through the SL4A, the PC use Python key algorithm "grafting" to the Android side, in addition,When implementing, call the camera module that comes with Android,the photo acquisition method to change the phone camera and take pictures to get photos through the phone, and then face recognition.

Figure 2.1 is a flowchart of the face recognition. 


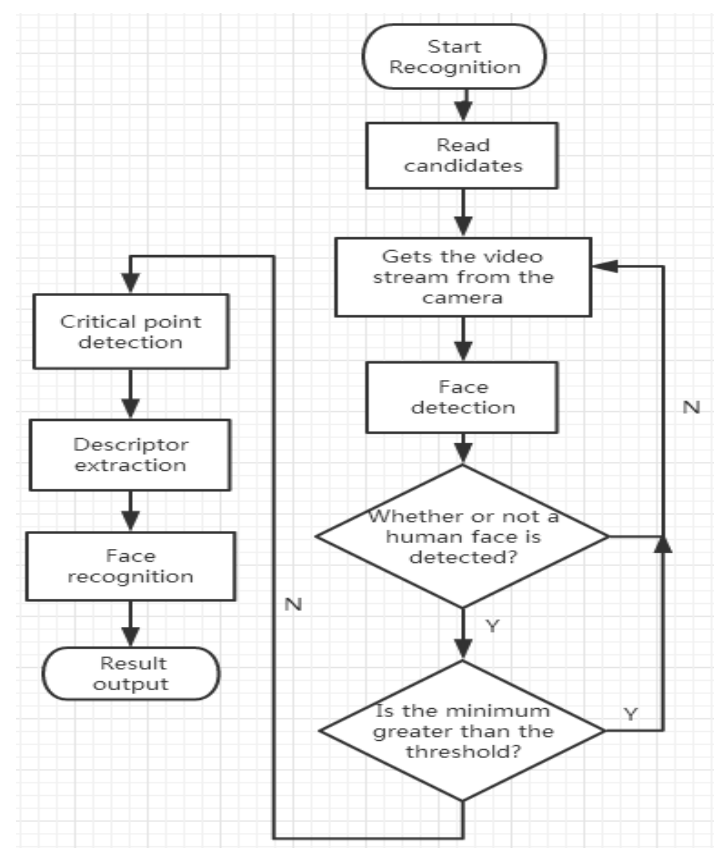

Figure 2.1 The flowchart of the face recognition.

\section{Experiment And Test}

The system is mainly based on the deep residual network to realize face recognition, through using the python interface of dlib to call the residual model for face recognition, the recognition accuracy of the model is more than 96\%,Finally, the experimental results are displayed on the PC side and the Android side, and the purpose of the test is to check whether the face can be detected and compared with the candidate folders, and get the correct results.

\subsection{The Implementation of PC}

The completion of PC, using the camera to capture the face in real time, saves the process of collecting and testing the pictures.The final recognition results are fed back to the user by adding sound peripherals, making the whole recognition process more humanized.Face image captured by the camera and the face detection and key point description is shown in Figure 3.1.

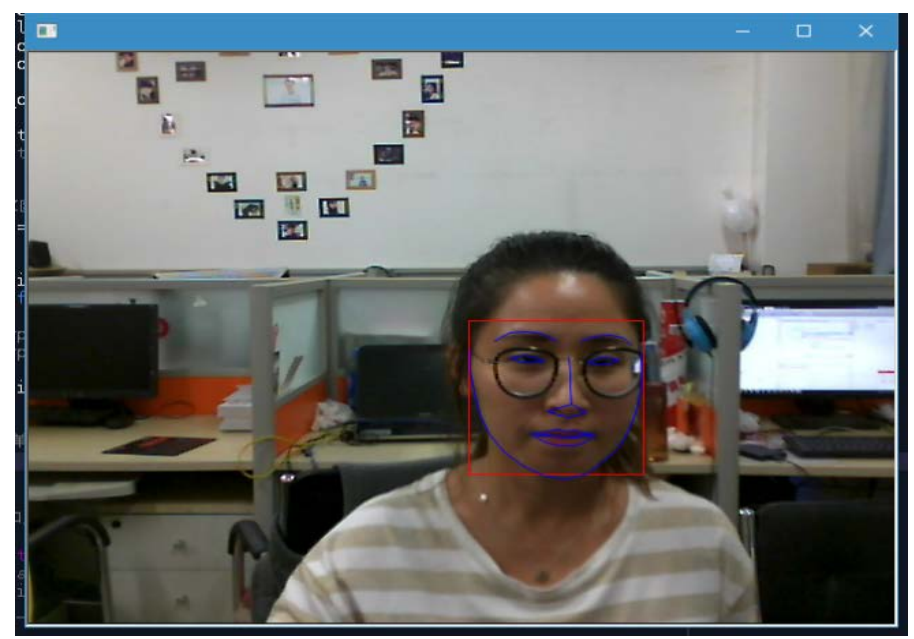

Figure 3.1 Face graph captured by the camera.

As can be seen from Figure 3.1,the user photos captured by the camera, and the residual model is called to detect and mark the key points. 
According to the previous presentation, the candidate folders are stored with seven candidate faces. Figure 3.2 is the face recognition to find a candidate Euclidean distance line chart, As shown in Figure 3.2 below ,abscissa represents the number of seven candidates, and the ordinate represents the Euclidean Distance value, the smaller the value indicates the higher the similarity of the candidate figure.

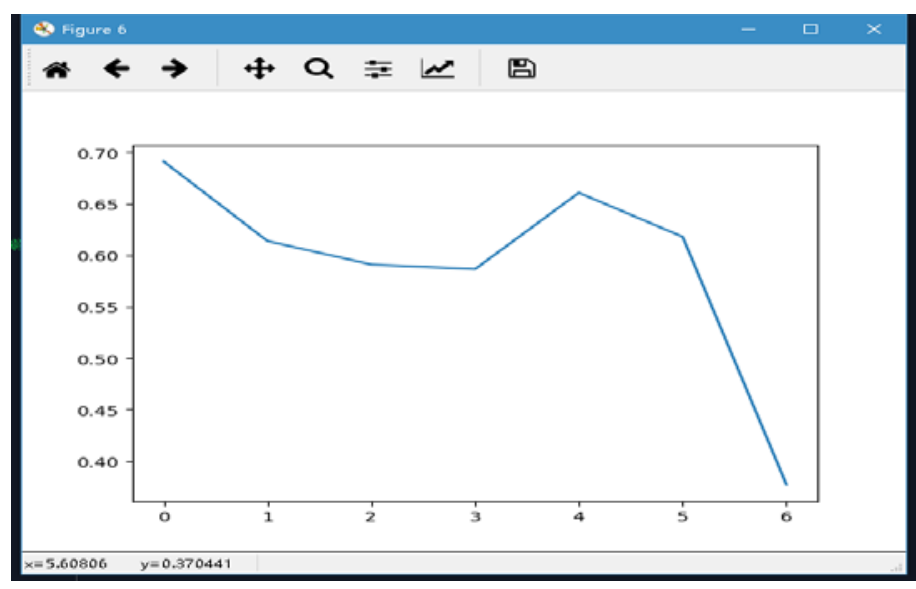

Figure 3.2 Euclidean distance line chart.

As shown in the above figure3.2, it can be seen that the Euclidean distance of No.6 is the smallest, And then compare the value of the European distance with accuracy of the variable, the accuracy value of 0.4, from Figure 3.2, the European distance value is less than the precision value, then determine the correct identification.According to candidate list candidate = [' Zhao Hongwei',' Li xing',' puppy Song Yifan',' jiangyouliang',' zhaihong',' Xu Xiaoli',' Liang Juan' ],It can be seen that No.6 candidate matches the test image successfully.

\subsection{The Implementation of Android}

The implementation of mobile terminal face recognition, call android own camera module, can real-time face detection and recognition, the Android end to meet the user to carry convenient, easy to operate.The realization of the Android side is shown in Figure 3.3, the results directly displayed in the bottom of the screen.

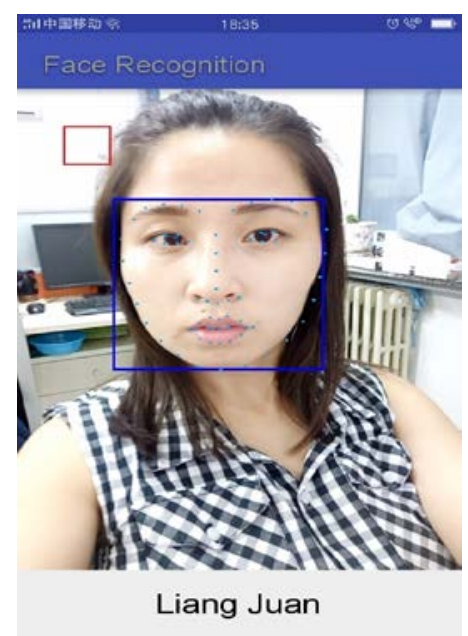

Figure 3.3 The completion of Android.

\section{Conclusion}

In this paper, face recognition based on the depth residual model is implemented in the PC and Android implementations, The PC side added peripheral devices such as camera and audio to make the recognition experience more smooth and comfortable, the Android side can make up for the 
inconvenience carrying by the PC terminal, can meet the different needs of users, provide security for users' life.This system may also be embedded in other terminals, such as embedded in the vehicle system, to prevent dangerous traffic, can also be used for some safety requirements of the high index of monitoring and real-time warning, greatly reduced the operation of human and material resources.

However, due to the recognition process by the light, photo quality, human attire, shelter and so on, we should further study and improve the implementation of the details of the study, look forward to the study have better performance and effectiveness.

\section{Acknowledgements}

The corresponding author is Zhao Hongwei. The authors are grateful to the anonymous reviewers for their insightful comments which have certainly improved this paper.

This work was supported in part by the Jilin province development and Reform Commission Special industrial innovation 2016C035 and State Key Laboratory of applied optics.

\section{References}

[1] Huihong Chen,Shiming Liu,Yaomin Hu.(2017) Face Recognition Technology Analysis and System Architecture . New Industrialization,2.

[2] Su N,Wu B,Xu W,et al.(2016)The Comprehensive Technology Development of Face Recognition. Journal of Information Security Research .

[3] Shu Zheng. (2017) Under the Shared Economy Innovation and Break . Special Purpose Vehicle, 7.

[4] He K, Zhang X, Ren S, et al.(2015) Deep Residual Learning for Image Recognition ,770-778.

[5] He K, Zhang X, Ren S, et al.(2016) Identity Mappings in Deep Residual Networks,630-645.

[6] Jordan L, Greyling P. (2011)Introducing SL4A: The Scripting Layer for Android. 